\title{
Modelling Hysteresis Loops in Fe-Based Soft Magnetic Composites Using Takács Description
}

\author{
B. JAnkowski ${ }^{a, *}$, B. Ślusarek ${ }^{a}$, J. SzCZYGŁOWski ${ }^{b}$ AND K. ChWASTEK ${ }^{b}$ \\ ${ }^{a}$ Tele and Radio Research Institute, Ratuszowa 11, 03-450 Warszawa, Poland \\ ${ }^{b}$ Częstochowa University of Technology, al. Armii Krajowej 17, 42-201 Częstochowa, Poland
}

(Received February 2, 2015)

\begin{abstract}
The measurements and modeling of hysteresis loops of soft magnetic composites made of powder with different particle sizes and different densities were investigated. The Takács description that is based on hyperbolic tangent transformation has been applied in the consideration. The paper shows recorded hysteresis loops with the maximum flux density of $1.2 \mathrm{~T}$.
\end{abstract}

DOI: $10.12693 /$ APhysPolA.128.116

PACS: $75.40 . \mathrm{Mg}, 75.47 . \mathrm{Np}, 75.50 . \mathrm{Bb}$

\section{Introduction}

The mathematical approximation of hysteresis loops is one of the elementary problem of ferromagnetism. In the last century, a numerous of approaches have been developed, like the Preisach model [1], vector hysteresis based on the Stoner-Wohlfarth model [2] and the JilesAtherton model [3]. In turn, Takács in paper [4] describes a purely mathematical approximation of the saturation curve and the hysteresis loop based on the $T(x)$ function which is composed of tangent hyperbolic and linear functions. The Takács model has been examined as the tool for separation of magnetic phases in magnetic alloys [5]. Takács has conjectured on the introduction of the effective field into his description in paper [6]. The concept of the effective field has been introduced more than century ago by Weiss [7]. The application of this concept has allowed to introduce the influence of additional physical phenomena on hysteretic, i.e. the effect of eddy currents, temperature or the effect of stress. Extended Takcács model has been successfully used to approximation of a dynamic hysteresis loops of cores made of soft magnetic composites [8].

Powder metallurgy has recently attracted the interest of the scientific and engineering community involved in the development of electric machines. This fact is closely related to manufacturing of parts for electric devices. Furthermore, powder metallurgy is characterized by low production costs, low powder waste and ease of recycling.

The expanding interest in the introduction of Fe-based composite materials in electrical devices, such as electrical motors, is obviously connected with their properties. The main feature of these materials is that iron particles are insulated by a thin organic or inorganic coating. SMCs offer several advantages over laminated steel

* corresponding author; e-mail: bartosz.jankowski@itr.org.pl sheets, for example isotropic magnetic properties, the opportunity to tailor their physical properties to requirements, very low eddy current loss, relatively high resistivity and high magnetic permeability [9-11].

The magnetic properties of soft magnetic composites significantly depend on processing parameters, i.e. a compaction pressure, an annealing temperature, a particle size distribution and a kind of coating layer. Shokrollahi and Janghorban in paper [12] present the effect of compaction parameters and particle size on magnetic properties of soft magnetic composites. They have reported that magnetic properties depend on densities, residual stress, and properties of coating layer. It is clear that with increasing pressure during compaction, green density increases but also leads to formation of residual stress. Suitable heat treatments can improve magnetic properties as a result of stress relaxation.

Anhalt in paper [13] has discussed particle size dependence of magnetic properties in soft magnetic properties. Author proves that the coercivity $H_{\mathrm{c}}$ shows a linear increase with increased inverse particle diameter $1 / d$. In addition, the effective number of active domain walls per particle is increased with an increased particle diameter owing to the lower amount of defects and pinning places in composites containing large particles compared with smaller particles.

The effect of particle sizes and core densities on magnetic properties of soft magnetic composites was investigated according to results of hysteresis loop modeling.

\section{Takács description of hysteresis loops}

The Takács description is based on hyperbolic tangent transformation of loop coordinates [4]. The branches of major loops are built in the Takács model by offsetting the argument of hyperbolic tangent function. In this work, the coefficients occurring in the Takács description have been identified as the magnetization $M$ and the effective magnetic field $H_{\text {eff }}=H+\alpha M$, where $\alpha$ is the so-called Weiss coupling coefficient. 
The hysteresis loops may be modeled by Eq. (1):

$$
M=M_{\mathrm{S}} \tanh \left(\frac{H_{\mathrm{eff}} \mp H_{\mathrm{c}}}{a}\right) \pm b\left(H_{\mathrm{eff}}^{V}, M^{V}\right),
$$

where $M_{\mathrm{S}}$ is saturation magnetization, $H_{\mathrm{c}}$ is coercive field strength, $a$ is a normalization constant, $b$ is a parameter dependent on the vertex coordinate of hysteresis loop $\left(H_{\mathrm{eff}}^{V}, M^{V}\right)$, which can be written as

$$
\begin{aligned}
& b\left(H_{\mathrm{eff}}^{V}, M^{V}\right)=\frac{1}{2}\left[\tanh \left(\frac{H_{\mathrm{eff}}^{V}+H_{\mathrm{c}}}{a}\right)\right. \\
& \left.-\tanh \left(\frac{H_{\mathrm{eff}}^{V}-H_{\mathrm{c}}}{a}\right)\right] .
\end{aligned}
$$

\section{Materials and experimental details}

Specimens investigated in this study were produced from commercially available pure iron powder produced by water atomization techniques, with a special surface coating on each and every particle offered under the trade name of Somaloy 700 (Höganäs AB Company).

The research has examined magnetic properties of soft magnetic composites made of powders with a different particle size distribution. The mean particle size of powders were respectively equal to 63,124 and $188 \mu \mathrm{m}$. Two sets of specimens was analyzed in the investigation. The first set of measurements covered the specimens compacted under pressure of $800 \mathrm{MPa}$ and hardened at $500{ }^{\circ} \mathrm{C}$. In turn, the second set covered the specimens compacted under different pressure and hardened at $500{ }^{\circ} \mathrm{C}$. Specimens of the second set had the same density. The annealing temperature was applied in accordance with the manufacturer's recommendations.

Measurements of the DC hysteresis cycle according to IEC Standards 60404-4 (Methods of measurements of d.c. magnetic properties of magnetically soft materials) were conducted using the system AMH-20K-HS produced by Laboratorio Elettrofisico Walker LDJ Scientific. Measurements of hysteresis loops were taken by recording individual points at maximum flux density $B_{\mathrm{m}}=1.2 \mathrm{~T}$ for ring shaped cores with following dimension: external diameter of $55 \mathrm{~mm}$, internal diameter of $45 \mathrm{~mm}$ and height of $5 \mathrm{~mm}$.

The particle-size distribution was obtained by laser diffraction technology using Horiba LA-950 particle size analyzer. The particle morphology observation were carried out using a scanning electron microscope JSM-7600F produced by JEOL.

Estimation of coefficient have been conducted using non-linear regression basing on the generalized reduced gradient (GRC) method. The generalized reduced gradient method is incorporated into the spreadsheet Microsoft Excel (add-on function SOLVER). This tool was described and applied by Brown in papers [3, 4]. The fitness value was defined as the squared sum of errors between the model value and experiment in all points on the $M-H$ plane.

\section{Results and discussion}

Densities of prepared specimens are summarized in Table I. It is worth to noting that specimens with large particle sizes are more compressible which is resulting in higher densities.

TABLE I

Densities of specimens made of Somaloy 700 powder with different mean particle size.

\begin{tabular}{c|c|c|c}
\hline \hline \multirow{2}{*}{} & \multicolumn{3}{|c}{ Mean particle size $[\mu \mathrm{m}]$} \\
\cline { 2 - 4 } & 63 & 124 & 188 \\
\hline specimens with & \multicolumn{3}{|c}{$7.18 \pm 0.07 \mathrm{~g} / \mathrm{cm}^{3}$} \\
the same density & \multicolumn{3}{|c}{} \\
\hline specimens compacted & $7.18 \pm 0.07$ & $7.30 \pm 0.07$ & $7.32 \pm 0.07$ \\
under pressure of $800 \mathrm{MPa}$ & $\mathrm{g} / \mathrm{cm}^{3}$ & $\mathrm{~g} / \mathrm{cm}^{3}$ & $\mathrm{~g} / \mathrm{cm}^{3}$
\end{tabular}

Figures 1 depict the morphology of particles used in the study. The investigation conducted by using scanning electron microscope confirms the difference in particle size. The mean size of the first fraction is equal to $63 \mu \mathrm{m}$ and the most particles (90\%) are less than $86 \mu \mathrm{m}$. In the case of the second fraction, the mean size is equal to $124 \mu \mathrm{m}$ and the most particles are less than $180 \mu \mathrm{m}$. In turn, for third fraction, the mean size is equal to $188 \mu \mathrm{m}$ and the most particles are less than $245 \mu \mathrm{m}$. The standard deviation of the particle size distribution are equal to 34,39 and $62 \mu \mathrm{m}$ respectively for the first, second and third fraction.

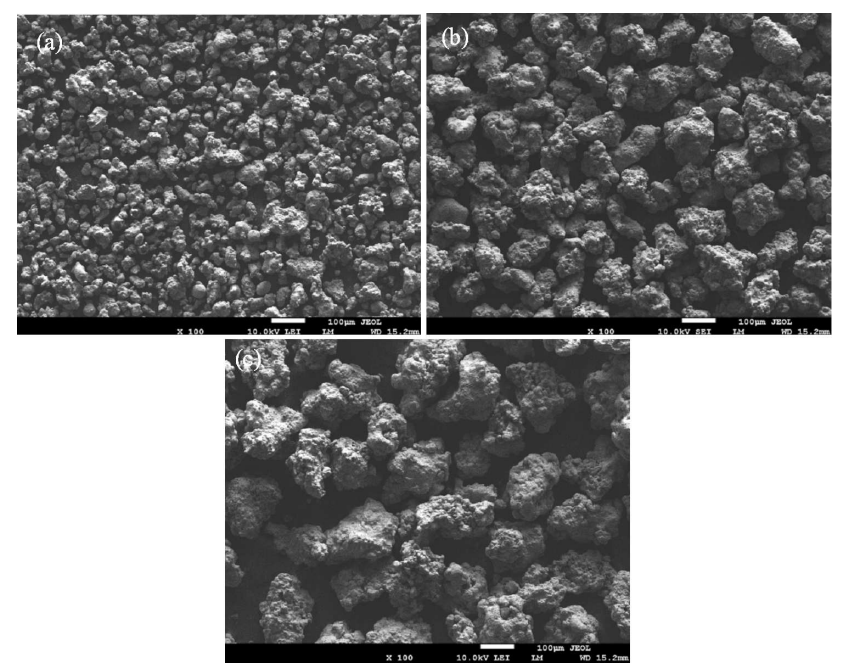

Fig. 1. Particles of Somaloy 500 powder: (a) $63 \mu \mathrm{m}$, (b) $124 \mu \mathrm{m}$, (c) $188 \mu \mathrm{m}$.

Table II presents the comparison of estimated coefficients $\alpha, M_{\mathrm{s}}, H_{\mathrm{c} 0}, a$. According to the results obtained for specimens with the same density we can observe that the coercive field and the slope of hysteresis loops decrease with increase of the mean particle sizes. Similarly to previous results, the slope of the hysteresis loops for specimens compacted under the same pressure of $800 \mathrm{MPa}$ decreases with increase of the mean 
particle sizes. In turn, the coercive field reveals a local minimum for the particle size with mean size of $124 \mu \mathrm{m}$ $\left(p=800 \mathrm{MPa}, \rho=7.30 \mathrm{~g} / \mathrm{cm}^{3}\right)$.

TABLE II

Coefficients of Takács description.

\begin{tabular}{c|c|c|c|c|c|c}
\hline \hline $\begin{array}{c}d \\
{[\mu \mathrm{m}]}\end{array}$ & $\begin{array}{c}\rho \\
{\left[\mathrm{g} / \mathrm{cm}^{3}\right]}\end{array}$ & $\begin{array}{c}p \\
{[\mathrm{MPa}]}\end{array}$ & $\begin{array}{c}\alpha \\
{\left[\times 10^{-5}\right]}\end{array}$ & $\begin{array}{c}M_{\mathrm{s}} \\
{[\mathrm{MA} / \mathrm{m}]}\end{array}$ & $\begin{array}{c}H_{\mathrm{c} 0} \\
{[\mathrm{~A} / \mathrm{m}]}\end{array}$ & $\begin{array}{c}a \\
{[\mathrm{~A} / \mathrm{m}]}\end{array}$ \\
\hline 188 & 7.32 & 800 & 1.3 & 0.946 & 273 & 1070 \\
124 & 7.30 & 800 & 3.4 & 0.948 & 267 & 1328 \\
63 & 7.18 & 800 & 7.4 & 0.945 & 333 & 2338 \\
124 & 7.18 & 550 & 9.1 & 0.945 & 327 & 1851 \\
188 & 7.18 & 500 & 6.3 & 0.944 & 309 & 1502
\end{tabular}

Figures 2, 3 and 4 depict the hysteresis loops of Fe-based composites cores made of powder with different particle sizes but compacted under the same pressure of $800 \mathrm{MPa}$.

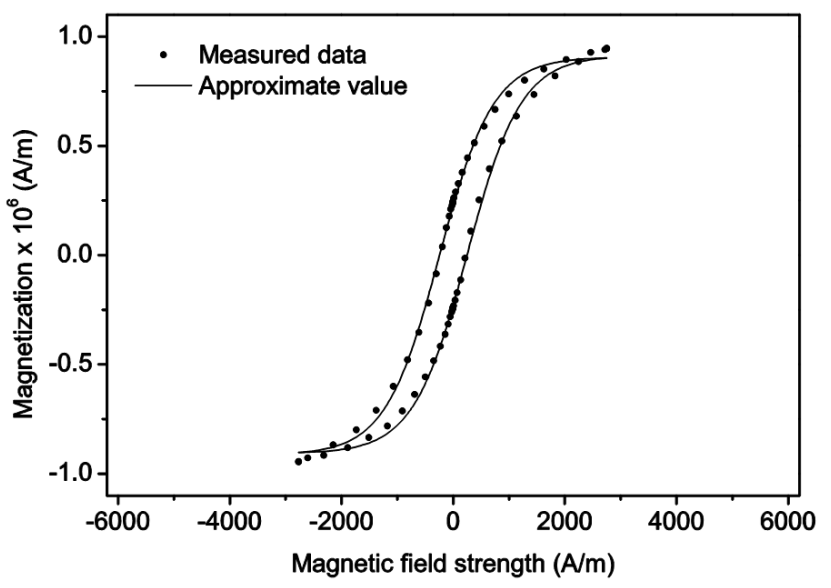

Fig. 2. Hysteresis loop of SMC made of powder with mean particle size $188 \mu \mathrm{m}$, density equal to $7.32 \mathrm{~g} / \mathrm{cm}^{3}$.

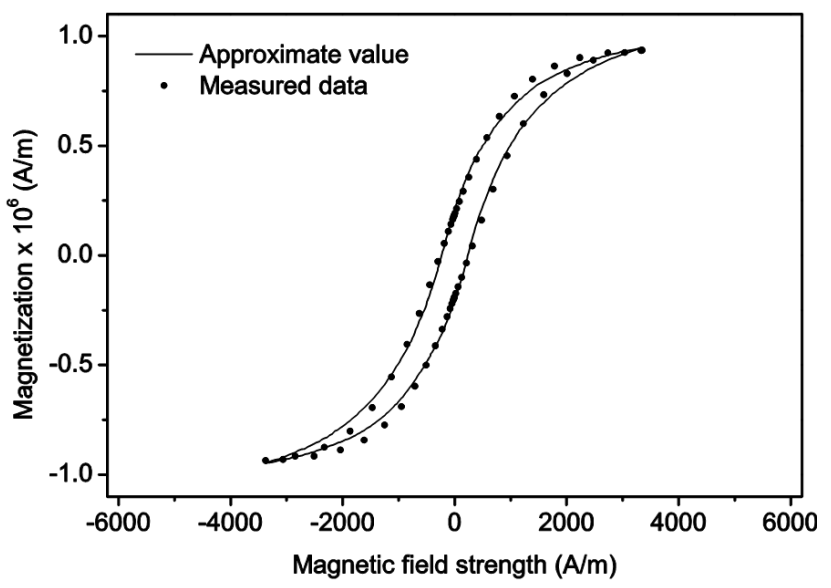

Fig. 3. Hysteresis loop of SMC made of powder with mean particle size $124 \mu \mathrm{m}$, density equal to $7.30 \mathrm{~g} / \mathrm{cm}^{3}$.

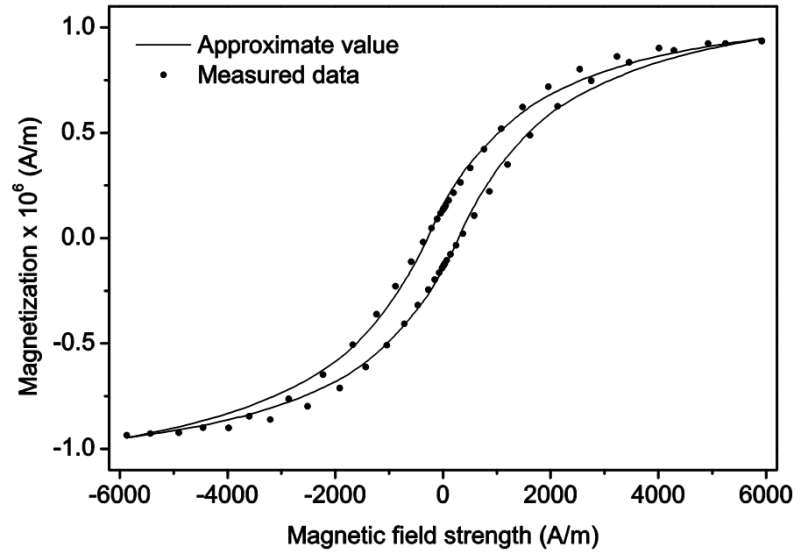

Fig. 4. Hysteresis loop of SMC made of powder with mean particle size $63 \mu \mathrm{m}$, density equal to $7.18 \mathrm{~g} / \mathrm{cm}^{3}$.

In turn, Figs. 4, 5, and 6 depict hysteresis loops of Fe-based soft magnetic composites made of powder with different particle sizes but the same densities. The modeled hysteresis loops exhibit good concordance with measured ones.

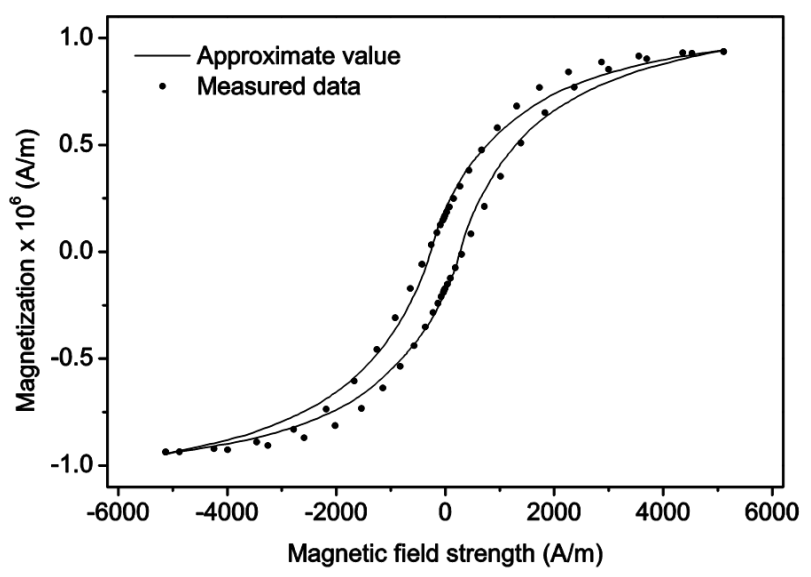

Fig. 5. As in Fig. 4, but for mean particle size $124 \mu \mathrm{m}$.

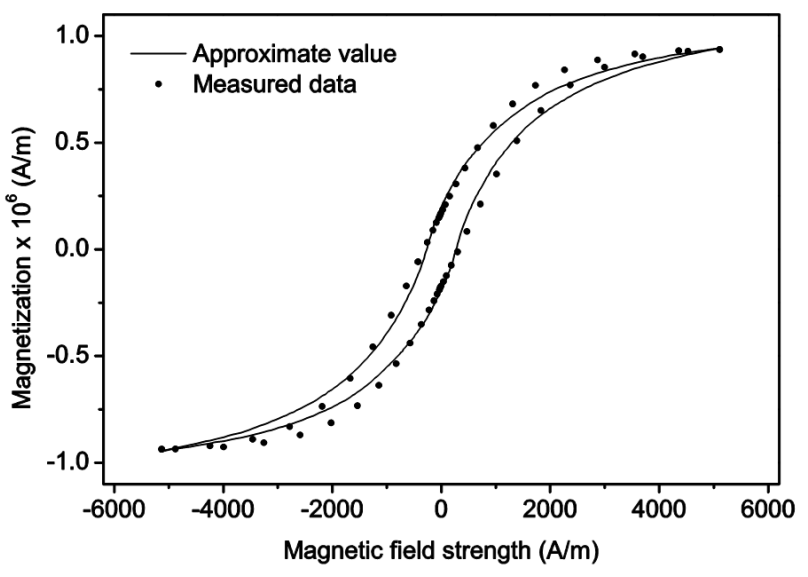

Fig. 6. As in Fig. 4, but for mean particle size $188 \mu \mathrm{m}$. 


\section{Conclusion}

In this paper the Takács approximation has been used to describe the hysteresis loops of cores made of soft magnetic composites. The study included the development of dependences between processing parameters (compaction pressure, particle sizes) and magnetic properties of soft magnetic composites. In order to obtain these relations, two set of specimens have been prepared under various compaction pressures and densities.

Experimental research and computation of hysteresis loops confirmed strong relationship between particle sizes and magnetic properties. It should be mentioned that the magnetization process depends greatly on the domain structure. Materials with large particle size (greater than $100 \mu \mathrm{m}$ ) have a complex structure of magnetic domains. The magnetization process in these materials takes place through the movement of magnetic walls and rotation of the magnetization vector and it is dominated by the first component. On the other hand, in the materials with a small size of particle (smaller than $100 \mu \mathrm{m}$ ), the rotation of the magnetization vector plays a dominant role $[14,15]$. The obtained results confirm mentioned above the physical relations of magnetization process. In the case of specimens with the same density, the coercivity represented by coefficient $H_{\mathrm{c} 0}$ and slope of hysteresis loop - coefficient $a$ decreases with increase of the mean particle size. These dependences prove that the magnetization process is easier for materials which contain large particles.

Another factor influencing the magnetic properties of soft magnetic composites is density which depends on compaction pressure. In the case of specimens compacted under the same pressure ( $p=800 \mathrm{MPa})$, the cores made of powder with mean particle size of 124 and $188 \mu \mathrm{m}$ exhibit very similar magnetic properties. Meanwhile, the core made of powder with mean particle size of $63 \mu \mathrm{m}$ reaches a much lower density compared to the other specimens which leads to significant differences in magnetic properties. This fact is related to the differences in the compressibility of powders with different sizes.

The proposed approximation of hysteresis loops was verified on the core specimens prepared under various processing parameters. The results of calculation exhibit good concordance with the experimental results which confirms suitability of the Takács description. Further research should focus on the influence of residual stress on magnetic properties of soft magnetic composites.

\section{References}

[1] F. Preisach, Z. Phys. 94, 5 (1935).

[2] E.C. Stoner, E.P. Wohlfarth, Philos. Trans. $R$. Soc. A 240, 826 (1948).

[3] D.C. Jiles, D.L. Atherton, J. Magn. Magn. Mater. 61, 1 (1986).

[4] J. Takács, COMPEL 20, 4 (2001).

[5] J. Takács, I. Mészáros, Physica B 403, 3137 (2008).

[6] J. Takács, COMPEL 24, 1180 (2005).

[7] P. Weiss, J. Phys. Archiv. 6, 1 (1907).

[8] B. Ślusarek, K. Chwastek, J. Szczygłowski, B. Jankowski, Solid State Phenom. 220-221, 652 (2015).

[9] A. Kordecki, B. Węgliński, J. Kaczmar, Powder Metall. 25, 4 (1982).

[10] H. Shokrollahi, K. Janghorban, J. Mater. Process. Technol. 189, 1 (2007).

[11] B. Ślusarek, Przeglad Elektrotechniczny 86, 16 (2010) (in Polish).

[12] H. Shokrollahi, K. Janghorban, Mater. Sci. Eng. B 134, 41 (2006).

[13] M. Anhalt, J. Magn. Magn. Mater. 320, e366 (2008).

[14] A. Kordecki, Dielectromagnetic Materials of Magnetic Cores of Electric Machines, Oficyna Wydawnicza Politechniki Wrocławskiej, Wrocław 1994.

[15] B. Jankowski, D. Kapelski, M. Karbowiak, M. Przybylski, B. Ślusarek, Powder Metall. 57, 2 (2014). 